\title{
1. Effect of Nanoslit Confinement on the Knotting Probability of 2 Circular DNA
}

\author{
${ }_{3}$ Liang Dai, $^{\dagger}$ Johan R. C. van der Maarel, ${ }^{\dagger, \ddagger}$ and Patrick S. Doyle ${ }^{* \dagger, \S}$ \\ $4^{\dagger}$ BioSystems and Micromechanics (BioSyM) IRG, Singapore-MIT Alliance for Research and Technology (SMART) Centre, 3 \\ 5 Science Drive 2, Republic of Singapore 117543 \\ ${ }_{6}{ }^{ \pm}$Department of Physics, National University of Singapore, 2 Science Drive 3, Republic of Singapore 117551 \\ $7{ }^{\$}$ Department of Chemical Engineering, Massachusetts Institute of Technology (MIT), Cambridge, Massachusetts 02139, United \\ 8 States
}

\section{S Supporting Information}

10 ABSTRACT: Monte Carlo simulations are used to study the 11 knotting probability of circular DNA confined in a slit. We 12 systematically vary the slit height, the width, and the contour 13 length of the DNA molecule. We find that the trend in 14 knotting probability with respect to slit height can be 15 monotonic or nonmonotonic, depending on the width and 16 contour length. The nonmonotonic trend is caused by two 17 competing factors: the increase of the effective persistence 18 length and the increase of segment density by slit confinement. 19 These factors are antagonistic, in the sense that the increase in 20 effective persistence length disfavors knot formation, whereas 21 the increase in segment density favors the knotting probability.

22 Our simulation results bring to light the importance of both chain length and width for slit-confined circular DNA and can be 23 used to guide future experiments which aim to produce populations of knotted DNA through cyclization or catalyzed double24 strand passage reactions in confinement.
${ }_{20}^{30} \mathrm{D}$ NA behavior in nanoconfinement is a burgeoning research area. The motivation has been multifold. First, 27 nanodevices with well-defined canonical geometries (e.g., tubes, 28 channels, and slits) provide platforms for fundamental studies 29 of the static and dynamic properties of polymers in confine30 ment. ${ }^{1-11}$ Second, the understanding of DNA behavior in 31 nanodevices can be applied to genome analysis. ${ }^{12-14}$ Third, in 32 vivo DNA routinely experiences a confined environment due to 33 quasi-stationary membranes and filaments in cells. Confine34 ment in turn can affect the biological function of DNA. For 35 example, DNA confined in a viral capsid experiences a high 36 pressure which can eject the DNA into bacteria during the 37 infection process. ${ }^{15}$

38 For a linear DNA chain, the effect of confinement on the 39 conformation has extensively been studied using theories, ${ }^{16-18}$ 40 simulations, ${ }^{19-21}$ and experiments. ${ }^{1-3}$ For a circular DNA 41 molecule, the effects of confinement are more complicated, due 42 to the interplay of geometrical confinement and topological 43 constraint (see ref 22 for a review). Such interplay may 44 contribute to the spatial organization of DNA in cells. ${ }^{23-25}$ 45 Circular DNA may also be knotted, ${ }^{26-29}$ which affects its statics 46 and dynamics. The formation of knots results from either 47 cyclization $^{30}$ or double-strand passage reactions catalyzed by 48 type II topoisomerase. ${ }^{31}$ The knotting probability, which is 49 defined as the fraction of knotted conformations from all so circular conformations, depends on the contour length and the effective diameter of the DNA molecule. ${ }^{26,30}$ In vivo, the 51 knotting probability is actively controlled by type II top- 52 oisomerases, because the knotting probability was found to be 53 as much as 80 times lower than at thermodynamic 54 equilibrium. ${ }^{31}$ The knotting probability was also observed to 55 be dramatically enhanced by confinement inside the capsid of 56 viruses. ${ }^{32} \mathrm{~A}$ virus capsid produces a nearly isotropic, spherical 57 confinement. Computer simulations show that the knotting 58 probability in spherical confinement monotonically increases 59 with increasing confinement. ${ }^{33}$

In contrast to spherical confinement, the knotting probability 61 in slit confinement (a form of uniaxial confinement) can be 62 nonmonotonic with increasing degree of confinement. Recent 63 computer simulations by Micheletti and Orlandini ${ }^{34}$ reveal that 64 the knotting probability is initially enhanced several fold by 65 weak and moderate slit confinement and then decreases toward 66 zero in strong slit confinement. In their simulations, the width 67 of the DNA chain is held constant at $2.5 \mathrm{~nm}$ and the DNA 68 length varied up to $4.8 \mu \mathrm{m}$. This chain width corresponds to 69 DNA at high ionic strength. In experiments or in vivo, the 70 effective chain width may be much larger than $2.5 \mathrm{~nm}$ due to 71 decreased screening in the electrostatic double layer.

72

Received: April 4, 2012

Accepted: May 25, 2012 
73 In our work, we perform Monte Carlo simulations of circular 74 DNA in slits and vary both the chain width and the contour 75 length. The protocol allows double-strand passage (crossing of 76 duplex), which simulates some aspects of type II topoisomerase 77 activity as well as the cyclization of linear chains. The knotting 78 probability calculated from simulations corresponds to the 79 fraction of knotted conformations in circular DNA which are 80 obtained from cyclization experiments. ${ }^{26,30}$ We find that the 81 effect of slit confinement on the knotting probability may be 82 nonmonotonic or monotonic, depending on chain width and 83 contour length. We show that this trend arises from a 84 competition between the effective persistence length and 85 segment density in slit confinement.

86 The simulation method is almost identical to the one we 87 recently used for linear DNA in a slit, ${ }^{20}$ except for some 88 modifications for the modeling of circular DNA. Here, we 89 briefly describe the simulation method. DNA is modeled as a 90 ring chain of $N$ beads connected by $N$ inextensible bonds of 91 length $l_{\mathrm{B}}$, corresponding to a contour length $L=N l_{\mathrm{B}}$. There are 92 three types of interactions in the simulations: the hard-core 93 repulsion between DNA beads, the hard-core repulsion 94 between DNA beads and slit walls, and the bending energy 95 between adjacent bonds. The hard-core diameter of the bead is 96 set to equal the bond length $l_{\mathrm{B}}$. The bead diameter is equivalent 97 to the chain width $w$, such that the number of beads equals $L /$ $98 w$. The values $w=5,10$, and $20 \mathrm{~nm}$ correspond to ionic 99 strengths of approximately 160,28 , and $6 \mathrm{mM}$, respectively. ${ }^{8}$ 100 The contour length is varied from $2 \mu \mathrm{m}(\sim 6 \mathrm{kbp})$ to $16 \mu \mathrm{m}$ 101 ( $\sim 7 \mathrm{kbp})$. For comparison, P4 phage DNA ( $\sim 10 \mathrm{kbp})$ is often 102 used to study the knotting in experiments ${ }^{30}$ and capsids. ${ }^{27,32}$ 103 The bending rigidity is set to reproduce a persistence length of $10450 \mathrm{~nm}$. We do not consider the twist energy of the circular 105 DNA, so that our model corresponds to a nicked open-circular 106 DNA (similar to what was performed in ref 34).

107 The simulation starts from an unknotted conformation. We 108 perform one crankshaft move in each Monte Carlo cycle. The 109 crankshaft move may switch an unknotted conformation to a 110 knotted conformation, or the converse. We do not prevent such 111 a move, to obtain the knotting probability, just like the 112 simulations by Micheletti and Orlandini. ${ }^{34}$ The DNA 113 conformations sampled by this method correspond to DNA 114 rings obtained by randomly cyclizing linear DNA. ${ }^{26,30}$ The 115 simulation usually reaches equilibrium in $10^{5}$ steps. In the 116 production run, we perform $10^{10}$ steps and record the 117 configuration every $10^{5}$ steps for data analysis. For the 118 estimation of the error in the calculation of knotting 119 probability, we divide the $10^{5}$ configurations into 10 bins, 120 calculate the average knotting probability in each bin, and then 121 calculate the standard deviation of these 10 values (see the 122 Supporting Information). The errors calculated in this way are 123 usually less than the symbol size in the figures, and most of our 124 figures do not show the error bars. We check whether or not 125 the circular DNA is knotted by the Alexander polynomial $\Delta(t)$, 126 following Vologodskii et l. $^{35}$ In the current study, we do not 127 classify the knot type.

f1 128 First, we present the simulation results in bulk. Figure 1 129 shows the knotting probability $k_{\text {bulk }}$ as a function of the contour 130 length. As the chain becomes longer, the knotting probability 131 becomes larger. Similar results have been reported in 132 experiments ${ }^{26}$ and computer simulations. ${ }^{30}$ In addition, as the 133 chain width $w$ becomes larger, the knotting probability 134 decreases. The dependence of $k_{\text {bulk }}$ on $w$ has been used to 135 infer the effective diameter of DNA from the knotting

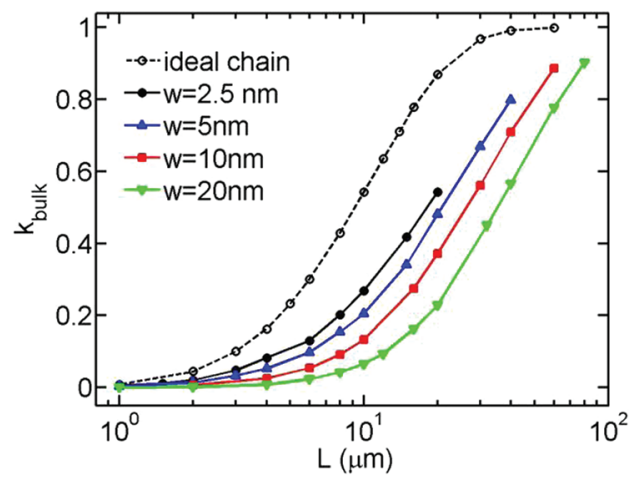

Figure 1. Knotting probability in bulk, $k_{\text {bulk }}$ as a function of the DNA contour length. Different colors (or symbols) correspond to different chain widths. $w=0$ corresponds to an ideal chain.

probability in the condition of different ionic strengths. ${ }^{30}$ The 136 chain width $w=0$ corresponds to an ideal chain in which 137 repulsion between DNA beads is turned off in the simulation. 138

Next, we proceed to the simulation results of a circular DNA 139 in a slit. Figure 2 shows the knotting probability $k_{\text {slit }}$ as a $140 \mathrm{f} 2$

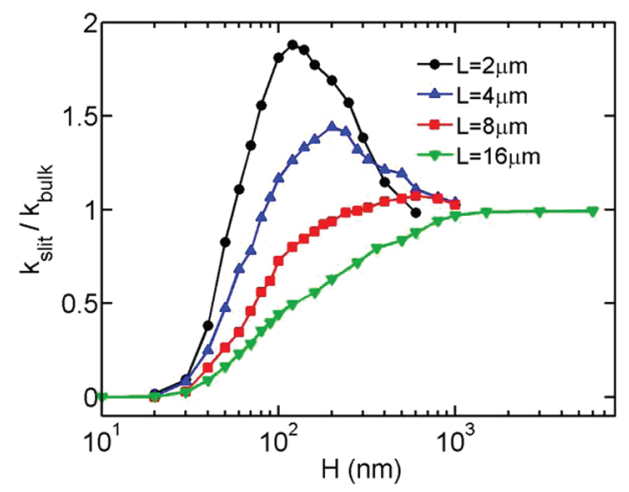

Figure 2. Normalized knotting probability as a function of the slit height. Different colors (or symbols) correspond to different contour length. The chain width is $10 \mathrm{~nm}$ for all curves.

function of the slit height. Different curves correspond to 141 simulations using different contour lengths, but with the same 142 chain width $w=10 \mathrm{~nm}$. The knotting probability is normalized 143 to the value in bulk. When the contour length is shorter than 144 about $8 \mu \mathrm{m}$, the slit confinement has a nonmonotonic effect on 145 the knotting probability. A similar trend has been observed in 146 the simulations by Micheletti and Orlandini. ${ }^{34,36}$ However, 147 when the contour length is $16 \mu \mathrm{m}$, increased confinement 148 always reduces the probability for knot formation. As the chain 149 length increases, the peak value of $k_{\text {slit }} / k_{\text {bulk }}$ becomes smaller, 150 and the peak position shifts to larger slit heights.

151

We now move on to study the effect of chain width. Figure $3 \quad 152 \mathrm{f} 3$ shows simulation results obtained by using different chain 153 widths, but with a fixed contour length of $8 \mu \mathrm{m}$. When the 154 chain width is less than or equal to $10 \mathrm{~nm}$, the knotting 155 probability is nonmonotonic. However, when the chain width is 156 $20 \mathrm{~nm}$, the trend becomes monotonic. As the chain width 157 increases, the peak value of $k_{\text {slit }} / k_{\text {bulk }}$ becomes smaller, and the 158 peak position shifts to larger slit heights. In the case of an ideal 159 chain (no excluded volume), $k_{\text {slit }}$ increases from $k_{\text {bulk }}$ in weak 160 and moderate confinement and slightly decreases from the peak 161 value in strong confinement. An ideal chain confined to a plane 162 can cross itself, and thus the knotting probability does not 163 


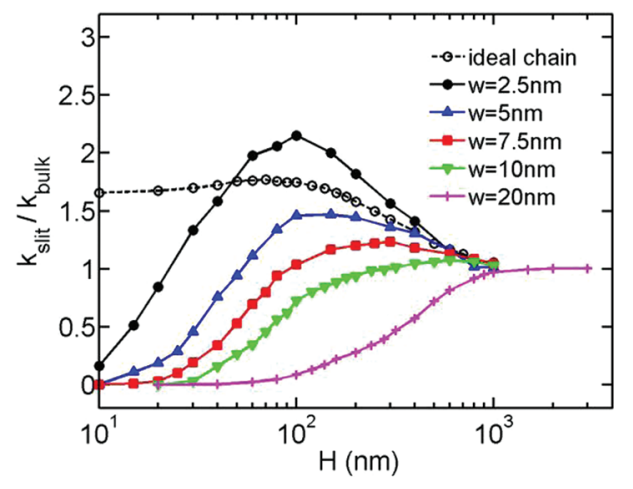

Figure 3. Normalized knotting probability as a function of the slit height. Different colors (or symbols) correspond to different chain widths. The contour length is $8 \mu \mathrm{m}$ for all curves.

164 approach zero when $H$ approaches zero, as shown by the 165 dashed line in Figure 3. Note that the ideal chain is not the 166 limiting case for a real chain with a vanishingly small width, 167 because a real chain can never cross itself when confined to a 168 plane, no matter how small the chain width becomes.

169 The peak positions in Figures 2 and 3 are related to the 170 confinement strength, that is, the ratio of the slit height to the 171 radius of gyration of a chain in bulk. For a thicker or longer 172 chain, the radius of gyration in bulk is larger, and so the peak 173 shifts to a larger slit height. In the Supporting Information, we 174 normalized the slit height to the radius of gyration of DNA in 175 bulk and replot Figures 2 and 3. The peak position in the 176 different curves occurs approximately when $H$ is on the order of 177 the bulk radius of gyration. In addition, we also show the peak 178 value and peak position as a function of the contour length and 179 width in the Supporting Information.

180 Combining the information in Figures 2 and 3, we can create 181 a plot (Figure 4) which generalizes the effect of slit

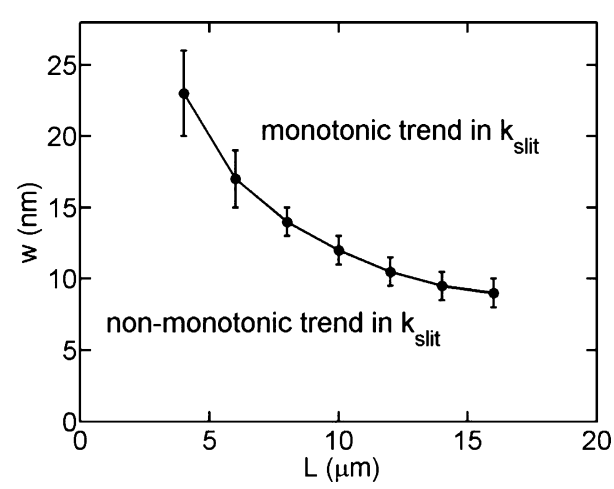

Figure 4. Diagram of the effect of slit confinement on the knotting probability as a function of the width and the contour length. The curve demarcates the boundary between the monotonic and the nonmonotonic $k_{\text {slit }}$ versus $H$ regions.

182 confinement on the knotting probability as a function of 183 chain width and contour length. The curve demarcates the 184 boundary of the nonmonotonic and monotonic regions. Above 185 the curve, that is, for long and thick chains, the slit confinement 186 monotonically decreases the knotting probability. Below the 187 curve, the knotting probability exhibits a nonmonotonic trend 188 when varying the slit height. Practically, it is difficult to obtain 189 the boundary for a very short or very long chain. For a very 190 short and thick chain (the left-hand end of the curve in Figure
4), the knotting probability is too small (less than 1\%) to 191 obtain the precise value. For a very long chain, the 192 computational time becomes impractical. It is expected that 193 for an infinitely long chain the critical chain width approaches 194 zero. This is because the knotting probability of an infinite long 195 chain in bulk is $1,{ }^{37}$ and the knotting probability in a slit cannot 196 exceed this value. With decreasing $L$, the critical chain width 197 increases more rapidly. However, the critical chain width 198 cannot increase to infinity, because it must be less than the 199 contour length. The nonmonotonic trend will occur for short 200 and thin chains, such as those used in the simulations by 201 Micheletti and Orlandini. ${ }^{34}$

202

To determine the mechanism for the nonmonotonic trend, 203 we have analyzed some other quantities of the DNA 204 conformation in the simulations. First, we consider the average 205 segment density $\bar{C}_{\text {seg }}$ as a function of the slit height. The 206 segment density is inversely proportional to the chain volume. 207 Here, the volume occupied by the chain is defined as the 208 product of the three eigenvalues of the radius of gyration 209 tensor, instead of the radius of gyration cubed $R_{\mathrm{g}}{ }^{3}$, because 210 DNA conformations in slits are significantly anisotropic. As 211 shown in Figure 5a, the segment density monotonically 212 f5
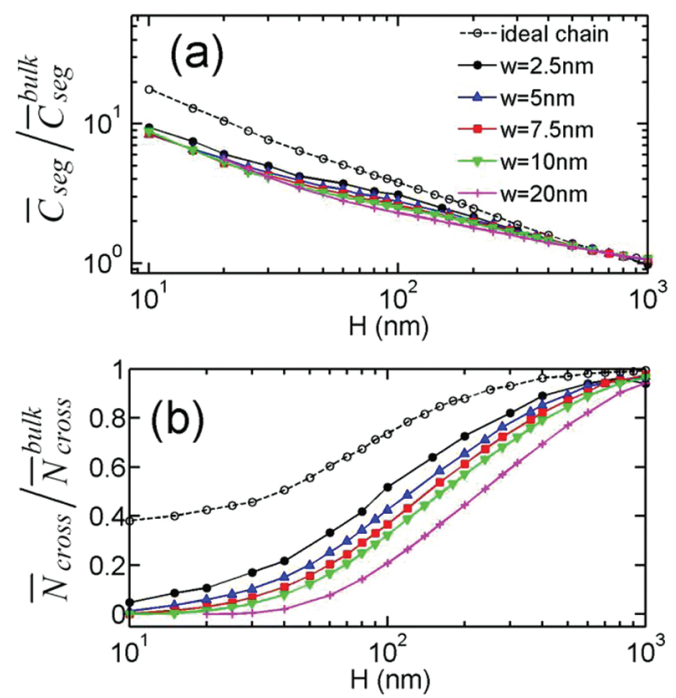

Figure 5. (a) Normalized DNA segment density as a function of the slit height. (b) Number of crossings as a function of the slit height. $\bar{N}_{\text {cross }}$ is the average number of crossings for the chain projection on a slit wall, which is normalized to the bulk value. Different symbols (colors) correspond to the simulations using different chain widths and the same contour length of $8 \mu \mathrm{m}$.

increases as the slit confinement becomes stronger. The 213 increase in segment density favors knot formation. Note that 214 if we use $R_{\mathrm{g}}^{3}$ to infer the segment density, we observe 215 nonmonotonic change of $R_{\mathrm{g}}{ }^{3}$ as a function of $H$, which agrees 216 with the results by Micheletti and Orlandini. ${ }^{34}$ However, $R_{\mathrm{g}}{ }^{3} 217$ does not accurately reflect the chain volume.

Next, we calculate the average number of self-crossing events 219 $\bar{N}_{\text {cross }}$ when we project the DNA chain on the slit wall, as shown 220 in Figure 5b. Note that $\bar{N}_{\text {cross }}$ here is not the number of 221 crossings used for the knot classification, because most of the 222 self-crossings can be removed by three types of Reidemeister 223 moves $^{38}$ in knot simplification. $\bar{N}_{\text {cross }}$ is normalized to the bulk 224 value $\bar{N}_{\text {cross. }}^{\text {bulk }}$ In bulk, we choose a random direction to project 225 DNA before counting the number of crossings. The values of 226 $\bar{N}_{\text {cross }}^{\text {bulk }}$ are $77.6,66.6,59.0,63.6$, and 39.5, when $w=2.5,5,7.5,227$ 
22810 , and $20 \mathrm{~nm}$, respectively. $\bar{N}_{\text {cross }}$ monotonically decreases as 229 the slit height decreases. Since $\bar{N}_{\text {cross }}$ is positively correlated 230 with the knotting probability, the decrease of $\bar{N}_{\text {cross }}$ by slit 231 confinement disfavors knot formation.

232 The nonmonotonic behavior of the knotting probability in 233 slit confinement appears to be the result of the competing 234 effects of $\bar{N}_{\text {cross }}$ and $\bar{C}_{\text {seg. }}$. However, as shown in Figure 4, the slit 235 confinement monotonically decreases the knotting probability 236 for a sufficiently thick chain or a sufficiently long chain. The 237 monotonic trend is most likely because the disfavoring factor of 238 knotting formation (decrease of $\bar{N}_{\text {cross }}$ ) overwhelms the 239 favoring factor (increase of $\bar{C}_{\text {seg }}$ ) for the whole range of 240 decreasing $H$ (increase in confinement). As shown in Figure $2415 \mathrm{a}, \mathrm{b}$, for a thicker chain, the favoring factor becomes weaker, 242 and the disfavoring factor becomes stronger. This may be the 243 reason for the monotonic trend when the chain width is 244 sufficiently large. In addition, the competition of the (dis)245 favoring factors should also depend on the value of $k_{\text {bulk }}$. For a 246 long chain, $k_{\text {bulk }}$ is large, and then the knotting probability is 247 relatively difficult to increase by slit confinement. As mentioned 248 above, the limiting case is that when the chain is infinitely long 249 with a maximal knotting probability of unity. Accordingly, for a 250 sufficiently long chain the knotting probability decreases 251 monotonically with increasing slit confinement.

252 The increase of $\bar{C}_{\text {seg }}$ by slit confinement is relatively easy to 253 understand. The decrease of $\bar{N}_{\text {cross }}$ by slit confinement is due to 254 the increase of the effective persistence length by slit 255 confinement. For an ideal chain, our previous simulations 256 show that the effective persistence length monotonically 257 increases when the slit height decreases. ${ }^{20}$ The effective 258 persistence length is extracted from the exponential decay of 259 the correlation of segment orientation. For a real chain, the 260 effective persistence length is also observed to be increased 261 greatly by slit confinement in strong confinement regime. ${ }^{39}$ In 262 addition, the excluded volume interaction prohibits the self263 crossing of a real chain when the slit height is less than the 264 chain width. As a result, for a real chain $\bar{N}_{\text {cross }}$ approaches zero 265 when $H$ approaches zero.

266 Our simulations demonstrate the interplay of spatial 267 confinement and topological states. Slit confinement increases 268 the segment density, which favors knot formation. On the other 269 hand, with decreasing slit height, the effective persistence length 270 of DNA increases from $L_{\mathrm{p}}$ in bulk to $2 L_{\mathrm{p}}$ in a plane. This 271 increase in orientation correlation length disfavors knot 272 formation. The excluded volume interaction is also of 273 importance, because it greatly decreases the knotting 274 probability in strong confinement. Overall, the results reveal 275 that all four lengths of the system, that is, persistence length, 276 chain width, contour length, and slit height, play essential roles 277 in determining the knotting probability. The competition of 278 different interactions gives rise to the nonmonotonic or 279 monotonic trend in the knotting probability. Different modes 280 of confinements (e.g., sphere, tube, slit) will in turn have 281 different effects on DNA behavior, because the competitions of 282 different interactions will vary. For instance, previous 283 experimental results reveal that the tube and slit confinements 284 have different effects on DNA compaction induced by 285 depletion. ${ }^{9,10}$ Spherical confinement monotonically increases 286 the knotting probability, ${ }^{33}$ which is different from the effect of 287 slit confinement. Thus, it would be interesting to examine the 288 effect of tube confinement on the knotting probability. Analysis 289 of the populations of special knot types in confinement is also an interesting topic, which has been explored by Micheletti and 290 Orlandini ${ }^{34}$ but only for a fixed chain width of $2.5 \mathrm{~nm}$. 291

Note that in our simulation the Monte Carlo moves allow for 292 a DNA segment to pass through another one. As mentioned 293 previously, the knotting probability calculated here thus 294 corresponds to the fraction of nontrivial knots in the DNA 295 rings cyclized from linear chains. ${ }^{26,30}$ The situation may also be 296 realized in the presence of type II topoisomerases. However, 297 type II topoisomerases change the distribution of different 298 topological states by a bias in double-strand passage reactions 299 through the dissipation of energy. ${ }^{31}$ In other words, in the 300 presence of type II topoisomerases the distribution in 301 topoisomers differs from the one obtained from random 302 passage reactions. Furthermore, in the absence of cutting 303 enzymes, the topology of circular DNA is of course 304 preserved. ${ }^{11,40}$ In this case, slit confinement has no effect on 305 the topological state of DNA.

The simulation results presented here can help to predict 307 experimental conditions to produce knotted DNA through 308 cyclization in confinement. The experiment in a slit rather than 309 in bulk should be more efficient to obtain knotted circular 310 DNA, provided the ionic strength is sufficiently high. For 311 example, in the case of $\lambda$-DNA with a contour length of $16 \mu \mathrm{m}, 312$ the ionic strength should be larger than $30 \mathrm{mM}$ (i.e., the 313 effective diameter ${ }^{8}$ of DNA is less than about $9 \mathrm{~nm}$ ) to increase 314 the knotting probability by slit confinement.

\section{ASSOCIATED CONTENT \\ Supporting Information}

Self-correlation of DNA topology in simulations; normalized 318 knotting probability as a function of the normalized slit height; 319 peak value and peak position of the knotting probability. This 320 material is available free of charge via the Internet at http:// 321 pubs.acs.org.

\section{AUTHOR INFORMATION}

\section{Corresponding Author}

*E-mail: pdoyle@mit.edu.

\section{Funding}

The authors declare no competing financial interest.

Notes

The authors declare no competing financial interest.

\section{ACKNOWLEDGMENTS}

330

This work is supported by the Singapore-MIT Alliance for 331 Research and Technology (SMART) and National Science 332 Foundation (NSF) Grant CBET-0852235. The authors thank 333 the Center for Computational Science and Engineering in 334 National University of Singapore for providing the computa- 335 tional resources. L.D. thanks A. V. Vologodskii for providing 336 the computer program of knot detection.

337

\section{REFERENCES}

(1) Reisner, W.; Morton, K. J.; Riehn, R.; Wang, Y. M.; Yu, Z.; Rosen, 339 M.; Sturm, J. C.; Chou, S. Y.; Frey, E.; Austin, R. H. Phys. Rev. Lett. 340 2005, 94, 196101.

(2) Bonthuis, D. J.; Meyer, C.; Stein, D.; Dekker, C. Phys. Rev. Lett. 342 2008, 101, 108303.

(3) Tang, J.; Levy, S. L.; Trahan, D. W.; Jones, J. J.; Craighead, H. G.; 344 Doyle, P. S. Macromolecules 2010, 43, 7368-7377.

(4) Zhang, C.; Zhang, F.; van Kan, J. A.; van der Maarel, J. R. C. J. 346 Chem. Phys. 2008, 128, 225109. 
348 (5) Kim, Y.; Kim, K. S.; Kounovsky, K. L.; Chang, R.; Jung, G. Y.; de 349 Pablo, J. J.; Jo, K.; Schwartz, D. C. Lab Chip 2011, 11, 1721-1729.

350 (6) Balducci, A.; Mao, P.; Han, J. Y.; Doyle, P. S. Macromolecules 351 2006, 39, 6273-6281.

352 (7) Hsieh, C. C.; Balducci, A.; Doyle, P. S. Macromolecules 2007, 40, 353 5196-5205.

354 (8) Hsieh, C. C.; Balducci, A.; Doyle, P. S. Nano Lett. 2008, 8, 16833551688.

356 (9) Jones, J. J.; van der Maarel, J. R.; Doyle, P. S. Nano Lett. 2011, 11, 357 5047-5053.

358 (10) Zhang, C.; Shao, P. G.; van Kan, J. A.; van der Maarel, J. R. C. 359 Proc. Natl. Acad. Sci. 2009, 106, 16651-16656.

360 (11) Lin, P. K.; Hsieh, C. C.; Chen, Y. L.; Chou, C. F. Macromolecules $3612012,45,2920-2927$.

362 (12) Tegenfeldt, J. O.; Prinz, C.; Cao, H.; Chou, S.; Reisner, W. W.; 363 Riehn, R.; Wang, Y. M.; Cox, E. C.; Sturm, J. C.; Silberzan, P.; Austin, 364 R. H. Proc. Natl. Acad. Sci. 2004, 101, 10979-10983.

365 (13) Jo, K.; Dhingra, D. M.; Odijk, T.; de Pablo, J. J.; Graham, M. D.; 366 Runnheim, R.; Forrest, D.; Schwartz, D. C. Proc. Natl. Acad. Sci. 2007, $367104,2673-2678$.

368 (14) Reisner, W.; Larsen, N. B.; Flyvbjerg, H.; Tegenfeldt, J. O.; 369 Kristensen, A. Proc. Natl. Acad. Sci. 2009, 106, 79-84.

370 (15) Gelbart, W. M.; Knobler, C. M. Science 2009, 323, 1682-1683.

371 (16) de Gennes, P. G., Scaling concepts in polymer physics; Cornell 372 University Press: Ithaca, NY, 1979.

373 (17) Odijk, T. Macromolecules 1983, 16, 1340-1344.

374 (18) Odijk, T. Phys. Rev. E 2008, 77, 060901.

375 (19) Wang, Y.; Tree, D. R.; Dorfman, K. D. Macromolecules 2011, 44, 376 6594-6604.

377 (20) Dai, L.; Jones, J. J.; van der Maarel, J. R. C.; Doyle, P. S. Soft 378 Matter 2012, 8, 2972-2982.

379 (21) Cifra, P. J. Chem. Phys. 2009, 131, 224903.

380 (22) Micheletti, C.; Marenduzzo, D.; Orlandini, E. Phys. Rep. Rev. 381 Sect. Phys. Lett. 2011, 504, 1-73.

382 (23) Jun, S.; Mulder, B. Proc. Natl. Acad. Sci. 2006, 103, 1238838312393.

384 (24) Fritsche, M.; Heermann, D. W. Soft Matter 2011, 7, 6906.

385 (25) Jung, Y.; Jeon, C.; Kim, J.; Jeong, H.; Jun, S.; Ha, B. Y. Soft 386 Matter 2012, 8, 2095.

387 (26) Shaw, S. Y.; Wang, J. C. Science 1993, 260, 533-536.

388 (27) Arsuaga, J.; Vazquez, M.; McGuirk, P.; Trigueros, S.; Sumners, 389 D.; Roca, J. Proc. Natl. Acad. Sci. 2005, 102, 9165-9169.

390 (28) Reith, D.; Cifra, P.; Stasiak, A.; Virnau, P. Nucleic Acids Res. 391 2012, DOI: $10.1093 /$ nar/gks 157.

392 (29) Marenduzzo, D.; Orlandini, E.; Stasiak, A.; Sumners, D.; 393 Tubiana, L.; Micheletti, C. Proc. Natl. Acad. Sci. 2009, 106, 2226939422274.

395 (30) Rybenkov, V. V.; Cozzarelli, N. R.; Vologodskii, A. V. Proc. Natl. 396 Acad. Sci. 1993, 90, 5307-5311.

397 (31) Rybenkov, V. V.; Ullsperger, C.; Vologodskii, A. V.; Cozzarelli, 398 N. R. Science 1997, 277, 690-693.

399 (32) Arsuaga, J.; Vazquez, M.; Trigueros, S.; Sumners, D.; Roca, J. 400 Proc. Natl. Acad. Sci. 2002, 99, 5373-5377.

401 (33) Micheletti, C.; Marenduzzo, D.; Orlandini, E.; Sumners, D. W. 402 Biophys. J. 2008, 95, 3591-3599.

403 (34) Micheletti, C.; Orlandini, E. Macromolecules 2012, 45, 21134042121

405 (35) Vologodskii, A. V.; Levene, S. D.; Klenin, K. V.; Frank406 Kamenetskii, M.; Cozzarelli, N. R. J. Mol. Biol. 1992, 227, 1224.

407 (36) Tesi, M. C.; Vanrensburg, E. J. J.; Orlandini, E.; Whittington, S. 408 G. J. Phys. A: Math. Gen. 1994, 27, 347-360.

409 (37) Sumners, D. W.; Whittington, S. G. J. Phys. A: Math. Gen. 1988, $41021,1689-1694$.

411 (38) Murasugi, K. Knot Theory and Its Applications; Birkhäuser: 412 Boston, 1996.

413 (39) Cifra, P.; Benkova, Z.; Bleha, T. Faraday Discuss. 2008, 139, 414 377-392.

415 (40) Strychalski, E. A.; Geist, J.; Gaitan, M.; Locascio, L. E.; Stavis, S. 416 M. Macromolecules 2012, 45, 1602-1611. 